\title{
Spatial and Temporal Scales of Neuronal Correlation in Primary Visual Cortex
}

\author{
Matthew A. Smith ${ }^{1}$ and Adam Kohn ${ }^{2,3}$ \\ ${ }^{1}$ Center for the Neural Basis of Cognition, Carnegie Mellon University, Pittsburgh, Pennsylvania 15213, ${ }^{2}$ Department of Neuroscience, Albert Einstein \\ College of Medicine, Bronx, New York 10461, and ${ }^{3}$ Center for Neural Science, New York University, New York, New York 10003
}

The spiking activity of cortical neurons is correlated. For instance, trial-to-trial fluctuations in response strength are shared between neurons, and spikes often occur synchronously. Understanding the properties and mechanisms that generate these forms of correlation is critical for determining their role in cortical processing. We therefore investigated the spatial extent and functional specificity of correlated spontaneous and evoked activity. Because feedforward, recurrent, and feedback pathways have distinct extents and specificity, we reasoned that these measurements could elucidate the contribution of each type of input. We recorded single unit activity with microelectrode arrays which allowed us to measure correlation in many hundreds of pairings, across a large range of spatial scales. Our data show that correlated evoked activity is generated by two mechanisms that link neurons with similar orientation preferences on different spatial scales: one with high temporal precision and a limited spatial extent $(\sim 3 \mathrm{~mm})$, and a second that gives rise to correlation on a slow time scale and extends as far as we were able to measure $(10 \mathrm{~mm})$. The former is consistent with common input provided by horizontal connections; the latter likely involves feedback from extrastriate cortex. Spontaneous activity was correlated over a similar spatial extent, but approximately twice as strongly as evoked activity. Visual stimuli thus caused a substantial decrease in correlation, particularly at response onset. These properties and the circuit mechanism they imply provide new constraints on the functional role that correlation may play in visual processing.

Key words: noise correlation; synchrony; population coding; multielectrode recordings; spontaneous activity; cross-correlogram; signal correlation; array

\section{Introduction}

The spiking activity of pairs of cortical neurons is correlated on a range of time scales. Two forms of correlation have received extensive theoretical and experimental attention. The first is shared trial-to-trial fluctuations in response strength (henceforth correlated variability), typically measured over hundreds of milliseconds. Correlated variability strongly influences population coding, but whether it is detrimental or beneficial depends on its properties, particularly its relationship to the tuning similarity of the neuronal pair (Zohary et al., 1994; Shadlen and Newsome, 1998; Abbott and Dayan, 1999; Averbeck et al., 2006). The variability of nearby neurons with similar tuning is known to be correlated (Zohary et al., 1994; Kohn and Smith, 2005), but visual stimuli activate neurons distributed over several millimeters of

Received June 11, 2008; revised Aug. 14, 2008; accepted 0ct. 4, 2008.

This work was supported by an National Institutes of Health National Research Service Award Fellowship to M.A.S. (National Eye Institute Grant EY015958), as well as National Institute of Mental Health Grant MH 64445 and National Science Foundation Computer \& Information Science \& Engineering Division of Information \& Intelligent Systems Grant 0713206 to Tai Sing Lee. We are grateful to Ryan Kelly, Amin Zandvakili, Xiaoxuan Jia, and Stephanie Wissig for assistance with data collection and technical expertise; to Wyeth Bair, Jason Samonds, and Simon Schultz for helpful advice and discussion; to Matt Harrison for providing software and assistance with the jitter correction method; and to Tai Sing Lee and J. Anthony Movshon for research support.

Correspondence should be addressed to Matthew A. Smith, at his present address: Department of Neuroscience and the Center for the Neural Basis of Cognition, University of Pittsburgh, 4400 Fifth Avenue, Mellon Institute, Room 115, Pittsburgh, PA 15213. E-mail: masmith@enbc.cmu.edu.

DOI:10.1523/JNEUROSCI.2929-08.2008

Copyright $\odot 2008$ Society for Neuroscience $\quad 0270-6474 / 08 / 2812591-13 \$ 15.00 / 0$ cortex (Van Essen et al., 1984; Tootell et al., 1988; Grinvald et al., 1994; Das and Gilbert, 1995). The extent and structure of correlated variability in such a distributed population is unknown. The second form of correlation-firing synchronized on a millisecond time scale, or synchrony-has a controversial history as a proposed code to bind the distributed representation of visual objects (Gray, 1999; Shadlen and Movshon, 1999), but is also known to affect rate-based population coding on brief time scales (Bair et al., 2001; Mazurek and Shadlen, 2002). The impact of synchrony on coding also depends on its extent and specificity in a distributed population, which are similarly unclear.

In addition to clarifying its effect on coding, measuring the structure of correlation is a way to elucidate the underlying mechanisms. Correlation arises from shared excitatory and inhibitory input (Moore et al., 1970; Lytton and Sejnowski, 1991; Morita et al., 2008), either from other stimulus-driven neurons or from ongoing activity (Arieli et al., 1996; Tsodyks et al., 1999; Chiu and Weliky, 2001; Kenet et al., 2003; Fiser et al., 2004). The circuitry relaying this shared input, however, is unclear. In primary visual cortex (V1), there are three obvious sources: (1) feedforward, thalamocortical axons that extend tangentially in layer IV for $<1 \mathrm{~mm}$ (Blasdel and Lund, 1983); (2) recurrent, horizontal connections that target neurons with similar orientation preference over distances of several millimeters but are locally $(<0.5$ $\mathrm{mm}$ ) nonspecific (Gilbert and Wiesel, 1983, 1989; Ts'o et al., 1986; Malach et al., 1993; Bosking et al., 1997); and (3) feedback 
A
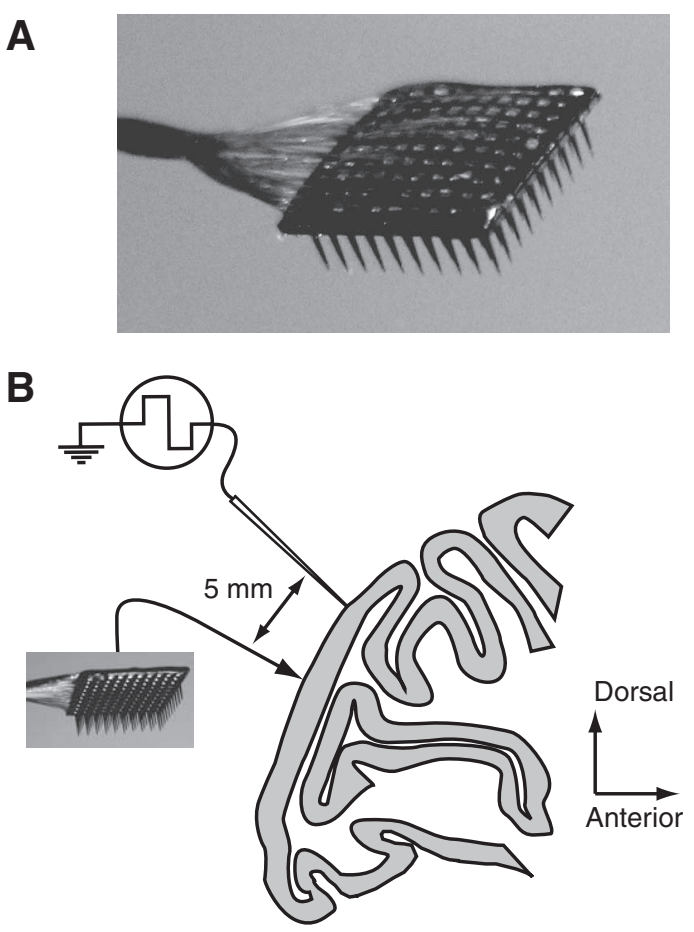

Figure 1. Experimental methods. $A$, A photograph of the array. The $10 \times 10$ grid of silicone microelectrodes had a $400 \mu \mathrm{m}$ spacing, $1.0 \mathrm{~mm}$ length, and was inserted $0.6 \mathrm{~mm}$ into cortex. $\boldsymbol{B}$, A diagram of the recording arrangement on the operculum of $\mathrm{V} 1$, shown in a sagittal section of macaque cortex. In two experiments, we simultaneously recorded from a group of seven linearly arranged microelectrodes, separated by at least $5 \mathrm{~mm}$ from the array.

connections from extrastriate cortex, which are far-reaching and weakly clustered (Angelucci et al., 2002; Shmuel et al., 2005) (but see Stettler et al., 2002). Because each of these pathways has a distinct spatial extent and functional specificity, measuring correlation structure should elucidate the relative contribution of each source (see also Seriès et al., 2004).

To determine the structure of correlation in a distributed population, we recorded from single neurons in V1 of macaque monkeys, with several innovations compared with previous studies. First, we used an array of 100 microelectrodes (Fig. $1 A$ ) to record from tens of neurons simultaneously, allowing us to compute correlation between many pairings separated by a range of distances and providing substantial power to detect even subtle trends. Second, we used a standard set of stimuli rather than optimizing for each pair of cells, providing more unbiased measurements of correlation. Finally, we used a recently developed analysis for isolating synchronous spiking from slower covariations in firing rate.

We find that correlation of evoked activity has a distinct spatial extent, depending on the time scale on which it is measured. Synchrony is evident between nearby neurons (separated by 3 $\mathrm{mm}$ or less) and is stronger between cells with similar orientation tuning, suggesting a prominent contribution from horizontal cortical connections. Correlated variability, on the other hand, decreases slowly with distance and is still significant between neurons separated by 10 millimeters, suggesting an important contribution from feedback connections. Correlation of spontaneous activity has similar spatial properties but is approximately twice as strong as that of evoked activity. These properties, and the circuit mechanisms that they imply, place new constraints on the functional role of correlation in visual processing.

\section{Materials and Methods}

We made seven array implants in seven hemispheres of four cynomolgus (Macaca fascicularis) adult male monkeys and one pig-tailed (Macaca nemestrina) adult male monkey. The animals ranged in weight from 3.6 to $5.0 \mathrm{~kg}$.

The techniques we use to record from the visual cortex of anesthetized, paralyzed macaques have been described in detail previously (Cavanaugh et al., 2002). Briefly, animals were premedicated with atropine sulfate $(0.05 \mathrm{mg} / \mathrm{kg}$ ) and diazepam (Valium, $1.5 \mathrm{mg} / \mathrm{kg}$ ) $30 \mathrm{~min}$ before inducing anesthesia with ketamine $\mathrm{HCl}(10.0 \mathrm{mg} / \mathrm{kg})$. Anesthesia was maintained throughout the experiment by a continuous intravenous infusion of sufentanil citrate (typically $6-18 \mu \mathrm{g} / \mathrm{kg}$, adjusted as needed for each animal). To minimize eye movements, the animal was paralyzed with a continuous intravenous infusion of vecuronium bromide $(0.1 \mathrm{mg} / \mathrm{kg} / \mathrm{h})$. Vital signs (EEG, ECG, blood pressure, end-tidal $\mathrm{P}_{\mathrm{CO} 2}$, temperature, and lung pressure) were monitored continuously. The pupils were dilated with topical atropine and the corneas protected with gas-permeable hard contact lenses. We used supplementary lenses to bring the retinal image into focus by direct ophthalmoscopy. We later adjusted the refraction further to optimize the response of recorded units. Experiments typically lasted $4-5 \mathrm{~d}$. All experimental procedures complied with guidelines approved by the Albert Einstein College of Medicine of Yeshiva University and New York University Animal Welfare Committees.

We recorded neural activity using the Cyberkinetics "Utah" Array (Cyberkinetics Neurotechnology Systems) (Fig. 1A), using methods reported previously (Kelly et al., 2007). The array consists of a $10 \times 10$ grid of silicon microelectrodes ( $1 \mathrm{~mm}$ in length) spaced $400 \mu \mathrm{m}$ apart, thus covering $12.96 \mathrm{~mm}^{2}$. We placed the array flush to the surface of the brain and inserted it $0.6 \mathrm{~mm}$ into cortex using a pneumatic insertion device (Rousche and Normann, 1992), resulting in recordings confined mostly to layers $2-3$. The impedance of microelectrodes in the array ranged from 200 to $800 \mathrm{k} \Omega$ with an average of $400 \mathrm{k} \Omega$ (measured with a $1 \mathrm{kHz}$ sinusoidal current). Signals from each microelectrode were amplified and bandpass filtered $(250 \mathrm{~Hz}$ to $7.5 \mathrm{kHz})$. Waveform segments that exceeded a threshold (periodically adjusted using a multiple of the rms noise on each channel) were digitized $(30 \mathrm{kHz})$ and sorted off-line.

The array was implanted in a $10 \mathrm{~mm}$ diameter craniotomy, centered 10 $\mathrm{mm}$ lateral to the midline and $8-10 \mathrm{~mm}$ posterior to the lunate sulcus, where neuronal receptive fields are within $5^{\circ}$ of the fovea. We extended the craniotomy as necessary to position the recording equipment. In two cases, we recorded simultaneously with a group of seven linearly arranged ( $2 \mathrm{~mm}$ extent) platinum-tungsten microelectrodes or tetrodes (Thomas Recording), positioned so that the nearest electrode was $\sim 5$ $\mathrm{mm}$ anterior to one edge of the multielectrode array. In this configuration, the distances between these electrodes and the array ranged from $\sim 5$ to $10 \mathrm{~mm}$.

Spike sorting and analysis criteria. Waveform segments were sorted off-line with an automated sorting algorithm, which clustered similarly shaped waveforms using a competitive mixture decomposition method (Shoham et al., 2003). We refined the output of this algorithm by hand with custom time-amplitude window discrimination software (written in MATLAB; MathWorks) for each electrode, taking into account the waveform shape and interspike interval distribution. To quantify the quality of the recording, we computed the signal-to-noise ratio (SNR) of each candidate unit as the ratio of the average waveform amplitude to the SD of the waveform noise (Nordhausen et al., 1996; Suner et al., 2005; Kelly et al., 2007). Candidates which fell below a SNR of 2.75 were discarded as multiunit recordings. We also eliminated neurons for which the best grating stimulus did not evoke a response of at least 2 spikes/s (62 units removed), or for which the variance-to-mean response ratio exceeded 10 ( 1 unit removed). The remaining candidate waveforms were deemed to be single units of sufficient quality and visual responsiveness to warrant further analysis. This procedure yielded a total of 246 cells recorded from 202 distinct electrodes (20-47 neurons per implant). Each neuron was then paired with all of the other simultaneously recorded neurons, excluding pairs from the same electrode because of the difficulty of detecting simultaneous spikes at a single site. Two pairs were dropped from our analysis because their spike pattern resulted in arti- 
facts in the CCGs with large peaks $>100 \mathrm{~ms}$ away from the zero bin. With these restrictions, our final dataset consisted of 4488 pairs. We examined the effect of changing our criteria for SNR and response rate on our results, and found no substantive differences.

Visual stimuli. We displayed all visual stimuli at a resolution of $1024 \times$ 768 pixels and a video frame rate of $100 \mathrm{~Hz}$ or $120 \mathrm{~Hz}$ on a CRT monitor. We used look-up tables to correct for nonlinearities in the relation between input voltage and phosphor luminance in the monitor. Stimuli were generated with custom software based on OpenGL, running on an Apple Macintosh G4 computer (EXPO, written by Peter Lennie, University of Rochester) or on a Silicon Graphics workstation. The mean luminance of the display was $\sim 40 \mathrm{~cd} / \mathrm{m}^{2}$. All of the stimuli were presented in a circular aperture surrounded by a gray field of average luminance.

For each array, we mapped the receptive fields of many single neurons on a tangent screen by hand. We determined the location of a group of receptive fields for each eye separately, and used a front surface mirror to center these fields on a monitor placed $110 \mathrm{~cm}$ from the animal, where it subtended $20^{\circ}$ of visual angle. We then proceeded with experiments under computer control.

We presented full-contrast drifting sinusoidal gratings at 12 orientations spaced equally $\left(30^{\circ}\right)$. The spatial frequency $(1.3 \mathrm{cpd})$ and temporal frequency $(6.25 \mathrm{~Hz})$ values were chosen to correspond to the typical preference of parafoveal V1 neurons (De Valois et al., 1982; Foster et al., 1985; Smith et al., 2002). The position and size $\left(8-10^{\circ}\right)$ of the grating were sufficient to cover the receptive fields of all the neurons. Stimuli were presented binocularly, for $1.28 \mathrm{~s}$, and separated by $1.5 \mathrm{~s}$ intervals during which we presented an isoluminant gray screen. Stimulus orientation was block randomized, and each stimulus was presented 100-200 times.

Measures of correlation. A detailed discussion of the methods and equations we use to characterize correlation can be found in Kohn and Smith (2005). Here, we describe the measures in brief.

The $r_{\mathrm{sc}}$, or spike count correlation, is the Pearson correlation coefficient of the evoked spike counts of two cells to repeated presentations of a particular stimulus: it captures shared trial-to-trial variability. For each stimulus orientation, we normalized the response to a mean of zero and unit variance (Z-score), and calculated $r_{\mathrm{sc}}$ after combining responses to all stimuli. We removed trials on which the response of either neuron was $>3$ SDs different from its mean (Zohary et al., 1994) to avoid contamination by outlier responses. We used Fisher's $r$-to- $Z$ transformation before performing statistical evaluation of $r_{\mathrm{sc}}$ values:

$$
z=\frac{1}{2} \ln \left(\frac{1+r_{\mathrm{sc}}}{1-r_{\mathrm{sc}}}\right)
$$

To measure the relative spike timing of two neurons, we computed the spike train cross-correlogram (CCG) (Perkel et al., 1967) as:

$$
C C G(\tau)=\frac{\frac{1}{M} \sum_{i=1}^{M} \sum_{t=1}^{N} x_{1}^{i}(t) x_{2}^{i}(t+\tau)}{\theta(\tau) \sqrt{\lambda_{1} \lambda_{2}}},
$$

where $M$ is the number of trials, $N$ is the number of bins in the trial, $x_{1}^{i}$ and $x_{2}^{i}$ are the spike trains of the two neurons on trial $i, \tau$ is the time lag, and $\lambda_{1}$ and $\lambda_{2}$ are the mean firing rates of the two neurons. $\theta(\tau)$ is a triangular function which corrects for the amount of overlap in the two spike trains at each time lag. We normalized the CCG by the geometric mean spike rate because it is the most commonly used normalization and provides results that are most easily compared with spike count correlation [for further discussion, see Kohn and Smith (2005)]. We smoothed the corrected CCGs with a 5 ms kernel ([0.05 0.25 0.400 .250 .05$])$ before using them in further analysis. We computed a separate corrected, normalized CCG for each of the 12 stimulus orientations, and then took the mean value at each time lag to produce the average CCG for each pair.

We corrected the CCG of each pair and stimulus condition for slow temporal correlations, as well as stimulus locked correlations, using a jitter correction method (Harrison et al., 2007; Harrison and Geman, 2008) [see Maldonado et al. (2000), Furukawa and Middlebrooks (2002), and Shmiel et al. (2006) for related approaches]. The standard shuffle- corrected CCG, created by subtracting the expected value of CCGs produced from the same dataset but with nonsimultaneous trial pairings, removes correlations that are locked to the stimulus. However, the resulting CCG reflects correlation on a range of time scales, including precisely aligned spike times and slow covariations in firing rate. The jitter-corrected CCG is created by subtracting the expected value of CCGs produced from a resampled version of the original dataset with spike times randomly perturbed (jittered) within a small temporal window (the jitter window). The correction term is the average over all possible resamples of the original dataset (i.e., the true expected value), and is subtracted from the raw CCG. The resulting jitter-corrected CCG is normalized by the geometric mean rate as in Equation 2 above.

This method is shown schematically in Figure $2 A$. The data from each neuron is divided into bins based on the jitter window (indicated with dashed vertical lines), starting at the stimulus onset. For each spike on each trial, a new spike is chosen randomly with replacement from the set of all spikes in the same jitter bin on all of the trials. In this way, the spike count in each bin and the neuron's PSTH are preserved in the resampled data. In Figure $2 B$, a raw CCG (red line, normalized as in Equation 2) is shown for an example pair of neurons, averaged across all 12 orientations of the stimulus, together with the correction terms produced by trial shuffling (dashed line) and the jitter method (gray lines for different jitter windows). The corrected CCGs, shown in Figure $2 C$, are produced by subtracting the correction term from the raw CCG for each stimulus orientation, and then averaging the resulting 12 CCGs. The shuffle correction (dashed line) removes correlation locked to the stimulus, but preserves correlation on a broad range of time scales. Jitter correction (Fig. 2C, solid lines) removes correlation in the CCG on a time scale greater than the jitter window. Because it preserves the PSTH shape, jitter correction also removes any correlation due to stimulus-locked firing rate modulation. The advantage of this method is that it helps to disambiguate short- from long-temporal correlations in the CCG. In the example pair shown (Fig. 2C), the progressively smaller jitter windows remove more of the long time scale correlation between the neurons (the broad peak in the CCG) while preserving short time scale correlation (the narrow peak, still evident with a jitter window of $50 \mathrm{~ms}$ ). In the limit, shortening the jitter window removes all temporal correlation between neurons (data not shown). In our analysis, we used a jitter window of 50 $\mathrm{ms}$ (thus dividing the $1.28 \mathrm{~s}$ stimulus period into 256 bins, starting at stimulus onset), but our results were not sensitive to the size of this window within a broad range $(25-100 \mathrm{~ms})$.

To relate our measures of timing (CCG) and count $\left(r_{\mathrm{sc}}\right)$ correlation, we calculated the metric $r_{\text {ccg }}$ (introduced by Bair et al., 2001), which is the integral of the CCG divided by the geometric mean area of the autocorrelograms (ACG) of the two neurons:

$$
r_{\mathrm{CCG}}(t)=\frac{\sum_{\tau=-t}^{t} \operatorname{CCG}(\tau)}{\sqrt{\left(\sum_{\tau=-t}^{t} \mathrm{ACG}_{1}(\tau)\right)\left(\sum_{\tau=-t}^{t} \mathrm{ACG}_{2}(\tau)\right)}} .
$$

The CCG is calculated as shown in the numerator of Equation 2 and is corrected by the all-way shuffle predictor; ACGs are calculated similarly but by correlating each neuron's activity against itself. We calculated $r_{\mathrm{ccg}}$ over different windows of integration, from $1 \mathrm{~ms}$ up to the length of the trial $(1.28 \mathrm{~s})$, to reveal the time scale over which correlated variability arises.

Finally, we compared our measures of response correlation to the tuning similarity of the two neurons, which we calculated as the Pearson correlation between the mean response of each cell to each of the tested orientations (termed $\left.r_{\text {signal }}\right)$. For neurons with similar orientation tuning $r_{\text {signal }}$ is near 1 , for neurons with dissimilar tuning $r_{\text {signal }}$ approaches -1 .

Fisher information. To evaluate the impact of the correlation structure we observe on the accuracy of a population code, we calculated the Fisher information of a population of V1 neurons. We used the analytical expression for Fisher information, derived for Gaussian distributed variables (an approximation for neuronal response distributions):

$$
I(\theta)=f^{\prime}(\theta)^{T} Q^{-1}(\theta) f^{\prime}(\theta)+\frac{1}{2} \operatorname{Tr}\left[Q^{\prime}(\theta) Q^{-1}(\theta) Q^{\prime}(\theta) Q^{-1}(\theta)\right],
$$


where $Q^{\prime}(\theta)$ and $f^{\prime}(\theta)$ are the derivatives of the covariance matrix and the tuning curves with respect to orientation, respectively; $Q^{-1}(\theta)$ is the inverse of the covariance matrix; and $\mathrm{Tr}$ stands for the trace operation (Kay, 1993; Abbott and Dayan, 1999).

The tuning of each cell, $f(\theta)$, was defined as:

$$
f(\theta)=m+a e^{b\left(\cos \left(\theta-\theta_{\mathrm{PREF}}\right)-1\right)}
$$

with $m=3, a=30, b=7$ (for a tuning bandwidth of $52^{\circ}$ ). We created multiple pools of 100 neurons, with $\theta_{\text {PREF }}$ spaced equally across orientations, and pools separated from each other by $0.5 \mathrm{~mm}$.

The entries of the covariance matrix were defined as:

$$
Q_{i j}(\theta)= \begin{cases}f_{i}(\theta) & \text { when } i=j, \\ r_{\mathrm{sc}}^{i, j} \sqrt{f_{i}(\theta) f_{j}(\theta)} & \text { when } i \neq j .\end{cases}
$$

That is, the variance of each cell was set equal to its mean response [defined by $f(\theta)$ ]. To explore the impact of different correlation structures, we set $r_{\text {sc }}$ to zero (independent neurons), a fixed value $(0.176)$, or allowed $r_{\mathrm{sc}}$ to decay with increasing distance or difference in tuning or both, as defined by Equation 7 and the data presented below. When only the dependence on $r_{\text {signal }}$ was considered, the distance in Equation 7 was set to zero; similarly, when only the distance dependence of $r_{\mathrm{sc}}$ was considered, $r_{\mathrm{sig}}$ nal was set to zero.

Mean and error values. For all mean values below, the errors are reported as \pm 1 SEM.

\section{Results}

We recorded single unit neuronal activity from V1 in 7 array implants performed in 5 macaque monkeys (Fig. 1). We measured the correlated variability and synchrony of spontaneous activity and activity evoked by drifting sinusoidal gratings whose parameters were chosen based on typical V1 preferences. We presented each stimulus a large number of times (100200) to obtain accurate measurements of correlation and to record a sufficient number of spikes to compute spike-train crosscorrelograms (CCGs). Using an off-line spike sorting procedure (see Materials and Methods), we identified 246 well isolated single units on 202 distinct electrodes. We computed correlation by pairing each neuron with all cells recorded simultaneously (excluding those on the same electrode), yielding a total of 4488 pairs.

\section{Dependence of $\boldsymbol{r}_{\mathrm{sc}}$ on distance and tuning similarity}

Trial-to-trial fluctuations in response strength are shared by pairs of cells. We measured this correlated variability with the Pearson correlation of the evoked spike count (termed $r_{\mathrm{sc}}$ ) over a $1.28 \mathrm{~s}$ period of visual stimulation. This analysis is based simply on count statistics and ignores the temporal structure of the two spike trains. Across the entire population of 4488 pairs, the average value of $r_{\mathrm{sc}}$ was $0.176 \pm 0.002$, similar to previous measurements in V1 (Gawne et al., 1996; Reich et al., 2001; Kohn and
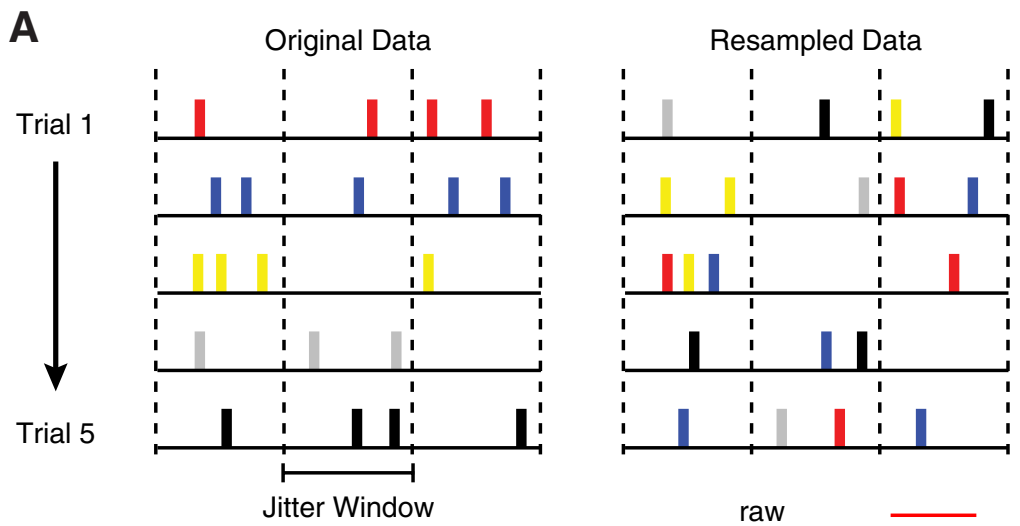

shuffle correction -.....

1000 ms jitter

$250 \mathrm{~ms}$ jitter

$50 \mathrm{~ms}$ jitter
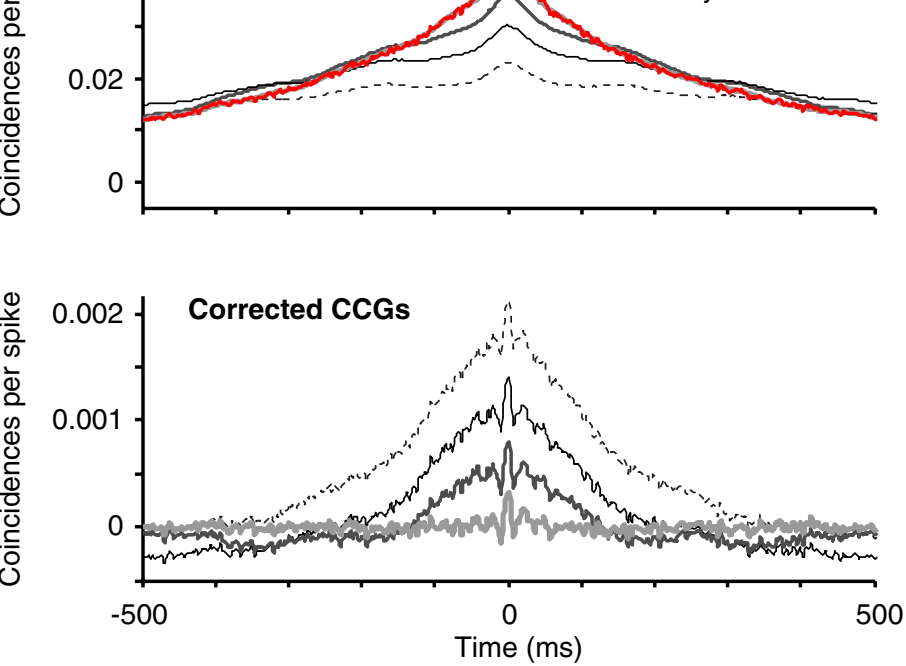

Time (ms)

Figure 2. Comparison of correction methods for CCGs. $A$, Diagram of the method used for CCG correction. Data from 5 simulated trials is shown on the left, with a different color labeling for the spikes from each trial. The trials were divided into bins based on he jitter window size (demarcated by the dashed vertical lines). From the original data, a raw CCG was computed. The original fall spikes in the same jitter bin on all trials. $\boldsymbol{B}$, Raw CCG and correction terms. From the original data, we computed a raw CCG (red for an example pair of neurons). The most common method of correcting (CGs is shuffling the trial labels, which and $1000 \mathrm{~ms}$, are shown here with gray lines (dark, thin lines represent the larger jitter windows). C. Corrected CCGs (subtracting the correction term from the raw $C(G)$. In the shuffle-corrected $C(G$ (dashed line), significant correlation is evident on both slow and fast time scales as broad and narrow peaks, respectively. Using jitter correction with large windows ( 250 and $1000 \mathrm{~ms}$ ), the broad peak is reduced but still evident. When the jitter window is small ( $50 \mathrm{~ms}$, thick gray line), the broad peak is removed and only the short time scale correlation remains.

Smith, 2005), MT/V5 (Zohary et al., 1994; Bair et al., 2001) and inferotemporal cortex (Gawne and Richmond, 1993).

We investigated how the strength of correlation depends on the distance between the neurons, estimated from the physical distance between the recording electrodes. We found a significant relationship between $r_{\mathrm{sc}}$ and distance $(r=-0.20, p<0.0001)$, with the activity of widely separated neurons being less correlated than nearby cells. This is illustrated in Figure $3 A$, in which we plot the decay of $r_{\mathrm{sc}}$ with distance for groups of pairs formed on the basis of their similarity in orientation tuning. Specifically, we measured tuning similarity using the metric $r_{\text {signal }}$ which ranges from -1 for dissimilar tuning to 1 for perfectly aligned tuning curves. Regardless of whether neurons had similar (thick line) or different (thin line) tuning, correlation decayed as the distance between neurons increased.

The maximum distance between electrodes of the array is 5.6 $\mathrm{mm}$, but we rarely recorded neurons separated by this distance. 

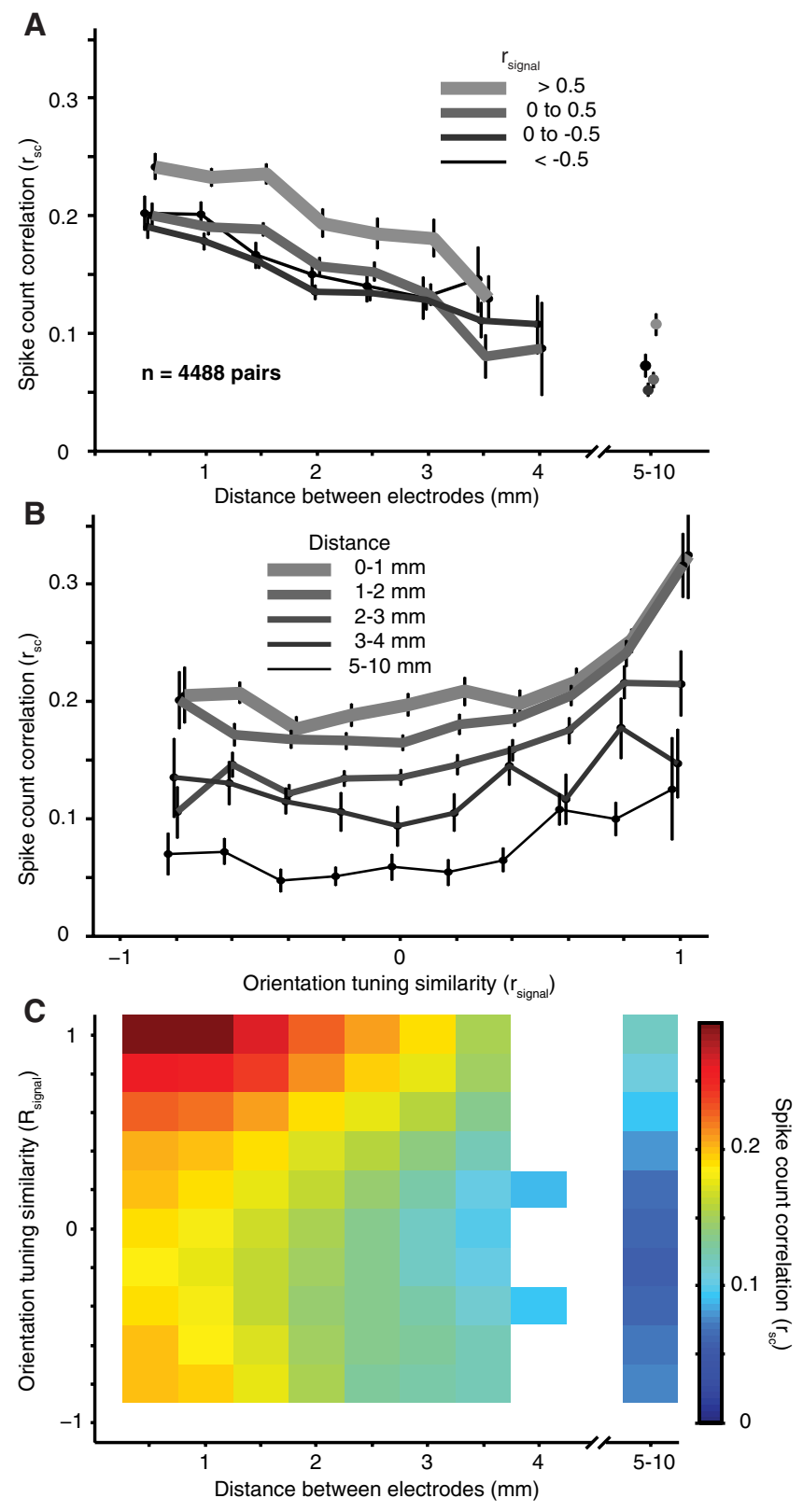

Figure 3. Dependence of $r_{\mathrm{sc}}$ on distance and tuning similarity. $\boldsymbol{A}$, The dependence of $r_{\mathrm{sc}}$ on distance for pairs grouped based on their orientation tuning similarity $\left(r_{\text {signal }}\right.$, thicker lines are for pairs with the most similar orientation tuning). The distance bins start at $0.25 \mathrm{~mm}$ and extend to $4.25 \mathrm{~mm}$ in $0.5 \mathrm{~mm}$ increments. The average of all the data are plotted at the center value of each bin. Data from dual-recording experiments (567 pairs) are shown with separate symbols to the right of the axis. Error bars on this and all subsequent plots indicate \pm 1 SEM; the points are shifted slightly from their true value along the abscissa for illustration purposes only. In this and all subsequent plots, only data points corresponding to 5 or more neuronal pairs are shown. $\boldsymbol{B}$, The dependence of $r_{\mathrm{sc}}$ on $r_{\text {signal }}$ for pairs grouped by their separation, thinner and darker lines are for more distant pairs. The average of all the data are plotted at the center value of each bin. $\boldsymbol{C}$, The dependence of $r_{\mathrm{sc}}$ on both distance and tuning similarity. The scale of the color plot is indicated by the bar at the right. The original data in this and all subsequent surface plots were smoothed with a two-dimensional Gaussian (SD of 1 bin).

At the maximum distance at which we could make reliable measurements $(4 \mathrm{~mm})$, we found that $r_{\mathrm{sc}}$ had declined to approximately half its peak value. From the array recordings, it is unclear whether this represents an asymptotic value or the truncation of a trend for $r_{\mathrm{sc}}$ to decay steadily toward zero for more widely separated neurons. This distinction is important for elucidating the spatial scale of the mechanisms that generate correlated variability.

To extend the range of our measurements, we performed additional recordings (in two array implants) in which we inserted a set of single electrodes or tetrodes into the superficial layers of V1 using an independent multielectrode system. These electrodes were placed at least $5 \mathrm{~mm}$ away from the nearest array electrode. By pairing neurons recorded with this system $(n=13)$ with those from the array, we were able to measure the value of $r_{\mathrm{sc}}$ over longer distances. Because we were unable to reconstruct the location of every electrode histologically, we collapsed the data from the 567 neuronal pairs recorded in this manner to a single bin which extended over distances ranging from 5 to $10 \mathrm{~mm}$. We noticed no trend for $r_{\mathrm{sc}}$ to decrease with distance within the 5-10 $\mathrm{mm}$ data before collapsing (in fact, there was a slight positive trend; $r=0.11, p=0.008$ ). Figure $3 A$ (rightmost points) shows the results of these measurements. At these large distances, the value of $r_{\text {sc }}$ was substantially weaker than that of most distant neurons sampled by the array but it remained significantly above zero $(0.064 \pm 0.004 ; p<0.0001)$. Thus, correlated variability is shared over a substantial swath of cortex, at least $10 \mathrm{~mm}$ in extent.

In addition to its dependence on distance, the value of $r_{\mathrm{sc}}$ reflected the similarity of the neurons' orientation tuning $\left(r_{\text {signal }}\right)$. Figure $3 B$ shows the dependence of $r_{\text {sc }}$ on $r_{\text {signal }}$ for pairs grouped by spatial separation. For neurons separated by either small (thickest lines) or large (thinnest lines) distances, there was a strong dependence of $r_{\mathrm{sc}}$ on tuning similarity: correlation was strongest for similarly tuned neurons and weakest between cells with opposite preferences. Thus, correlation on spatial scales extending up to $10 \mathrm{~mm}$ reflects circuitry that links neurons with similar preferences.

We summarize the relationship between $r_{\mathrm{sc}}, r_{\text {signal }}$, and distance in Figure 3C. The largest values of $r_{\mathrm{sc}}$ (red regions) are in the upper left corner of the plot, for neurons that are close together and have a similar preference for orientation. We found that the data of Figure $3 C$ (and the synchrony data presented below) were well fit by a product of two functions, a linear decay with distance and an exponential decay with $r_{\text {signal }}$ :

$$
r_{\mathrm{sc}}=[a-b(\text { distance })]^{+} e^{\frac{r_{\text {signal }-1}}{\tau}+c,}
$$

where []$^{+}$indicates that negative values of the linear term are set to 0 . The linear decay had an intercept $(a)$ of $0.225 \pm 0.026(95 \%$ confidence interval) and slope $(b)$ of $0.048 \mathrm{~mm}^{-1} \pm 0.008$. The exponential had a decay constant $(\tau)$ of $1.87 \pm 0.67$ (unitless). The baseline added to the product of these functions $(c)$ had a value of $0.09 \pm 0.03$. This function, with four parameters, accounted for $89.4 \%$ of the variance in the data ( 72 combinations of distance and $r_{\text {signal }}$ for the data recorded within the array).

\section{Time scales of correlation}

Correlated trial-to-trial variability can arise from shared input that generates tightly synchronized firing or activity that is more loosely coordinated in time. Our measurements of $r_{\mathrm{sc}}$ were made on a fixed time scale (the length of the trial) and ignored the relative temporal structure of the two spike trains. To extend these measurements, we calculated the metric $r_{\mathrm{ccg}}$, which is equal to the integral of the CCG normalized by the area under the autocorrelograms of the respective neurons (see Materials and Methods). When integrated over a time lag equal to the duration of the trial, $r_{\mathrm{ccg}}$ is mathematically equivalent to $r_{\mathrm{sc}}$ (Bair et al., 2001). Integrating over smaller windows reveals the strength of 

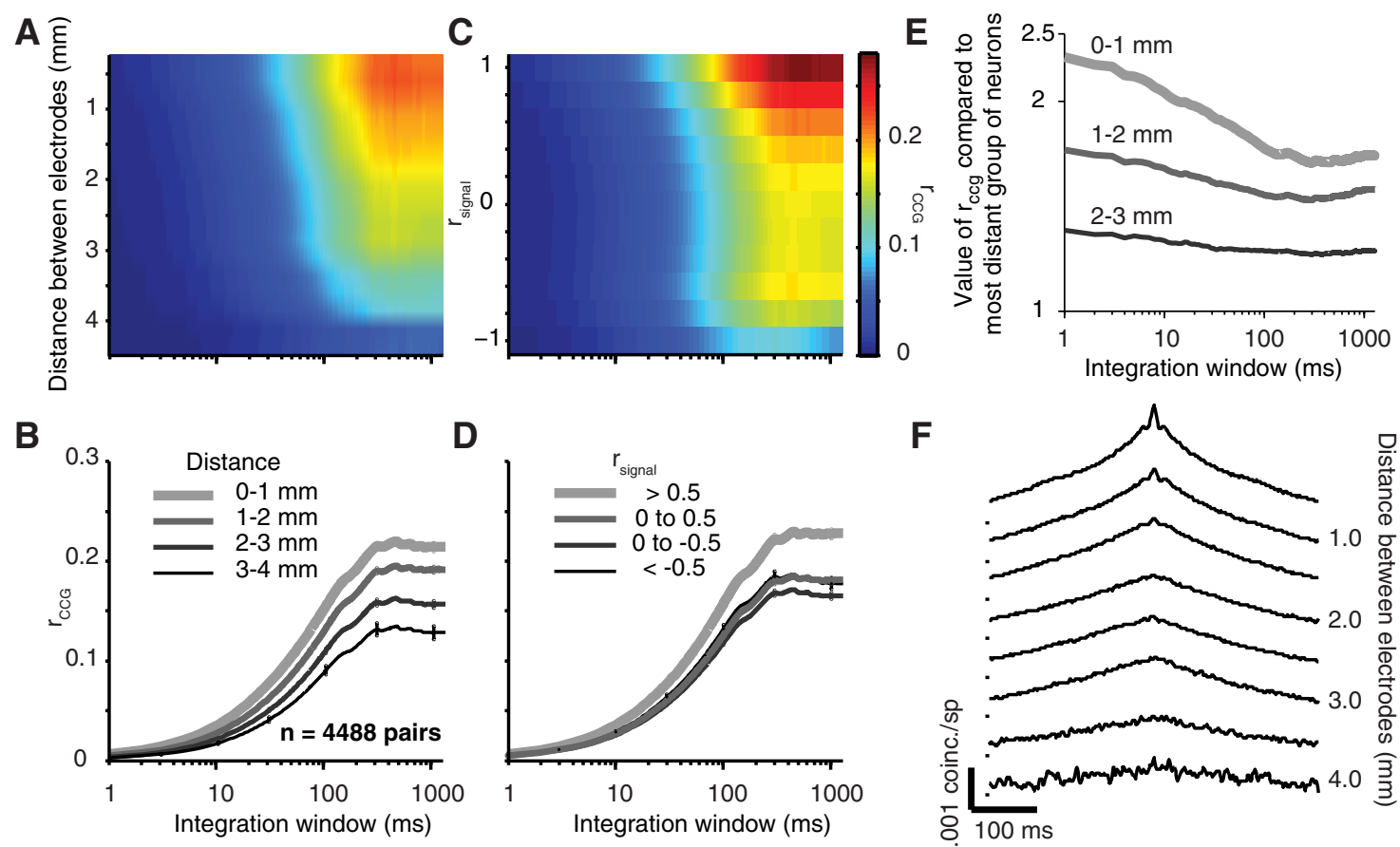

Figure 4. Effect of distance on the time scale of correlation. $A$, Surface plot showing the value of $r_{\text {ccg }}$ for different integration windows and distances between electrodes. The color scale is shown to the left of $\boldsymbol{E}$. $\boldsymbol{B}, r_{\text {ccg }}$ as a function of integration window for different distance groups (thinner and darker lines for more distant pairs). $\boldsymbol{C}$, Surface plot showing the value of $r_{\text {ccg }}$ for different integration windows and tuning similarity. The color scale is shown on the right. $\boldsymbol{D}, r_{\text {ccg }}$ as a function of integration window for different $r_{\text {signal }}$ groups (thinner and darker lines indicate pairs with less similar tuning preferences). $\boldsymbol{E}$, For neurons in three distance groups (thinner and darker lines are more distance), the value of $r_{\text {ccg }}$ is shown as a ratio to its value at the longest distance ( $3-4 \mathrm{~mm}$ ). $\boldsymbol{F}$, Average shuffle-corrected CCGs for pairs of neurons grouped by distance. The tick marks to the left of the CCGs indicate a value of 0 coinc./sp.

correlation arising from increasingly precise coordination of timing in the neurons' spike trains.

The value of $r_{\mathrm{ccg}}$, computed over a range of temporal integration windows and for neurons separated by different distances, is shown in Figure $4 A$. Cross sections of this surface (Fig. $4 B$ ) illustrate the behavior of $r_{\mathrm{ccg}}$ for four distance groups $(0.0-4.0 \mathrm{~mm}$ in $1.0 \mathrm{~mm}$ steps, as in Fig. $3 B$ ), with the thickest lines representing pairs separated by the smallest distance. At each distance, the strength of $r_{\text {ccg }}$ increases with the size of the integration window, up to a time scale of 200-300 ms where it reaches a plateau. The rightmost data point on each curve (arising for integration windows of $1.28 \mathrm{~s}$ ) is equivalent to $r_{\mathrm{sc}}$; comparing these endpoints for different distance groupings illustrates the previously described trend for $r_{\mathrm{sc}}$ to decrease for neurons that are more widely separated. This distance dependence holds across shorter time scales as well (discussed in more detail below). A similar analysis for the dependence of $r_{\text {ccg }}$ on tuning similarity is shown in Figure $4 C$, with the corresponding cross sections shown in Figure $4 D$. Neurons with similar orientation preferences (thick line) exhibited higher correlation across all time scales.

To test directly whether correlation decayed similarly across time scales, we normalized the $r_{\text {ccg }}$ curves of each distance group by the trend seen for the most widely separated neurons (Fig. $4 E$ ). That is, we divided each of the curves in Figure $4 B$ by the data shown by the thinnest line in that panel. These normalized values indicate, for each time scale, the scaling factor needed to correct for the distance dependence of correlation. If the decay of $r_{\mathrm{ccg}}$ with distance were independent of the integration window, these normalized traces would appear as a series of horizontal lines. Instead, the lines are sloped downward, particularly for integration windows $<100 \mathrm{~ms}$. This indicates that correlation arising from activity synchronized on time scales $<100 \mathrm{~ms}$ decays more rapidly with distance than correlation measured on longer time scales.

To confirm this conclusion with independent analysis, we computed shuffle-corrected CCGs (see Materials and Methods) for each pair. Precisely synchronized spiking results in a sharp peak in the CCG, while correlation on a longer time scale leads to a broad peak. The average CCGs, divided into 8 distance groups, are shown in Figure $4 F$. These CCGs also suggest the presence of two processes that decay with distance at different rates: a broad peak that decays slowly with distance; and a sharp peak, reflecting synchronous spiking, that decays more rapidly and is prominent only between pairs of neurons separated by $<2 \mathrm{~mm}$.

\section{Dependence of synchrony on distance and tuning similarity}

The synchrony seen in the shuffle-corrected CCGs (Fig. 4F) involves a sharp peak as well as a broader component which reflects more loosely coordinated activity. To study the precise synchrony in more detail, we turned to a novel analysis to isolate those events. Rather than correcting each CCG by a shuffle predictor (as in Fig. $4 F$ ), we subtracted a CCG computed from a jittered version of the original spike train (see Materials and Methods and Fig. 2). Briefly, the data from each trial was divided into bins based on the size of the jitter window. For each spike in each jitter bin, a new spike was chosen randomly, without replacement, from the set of all spikes in the same jitter bin on all trials. Thus, the spike count on each trial and overall PSTH shape were preserved. The correction term calculated from this resampled spike train removes stimulus-locked correlation (like the shift or shuffle predictor) but also eliminates all correlation occurring outside a temporal scale defined by the jitter window. This method thus allows us to study the behavior of synchrony without contamination from more loosely coordinated activity. 


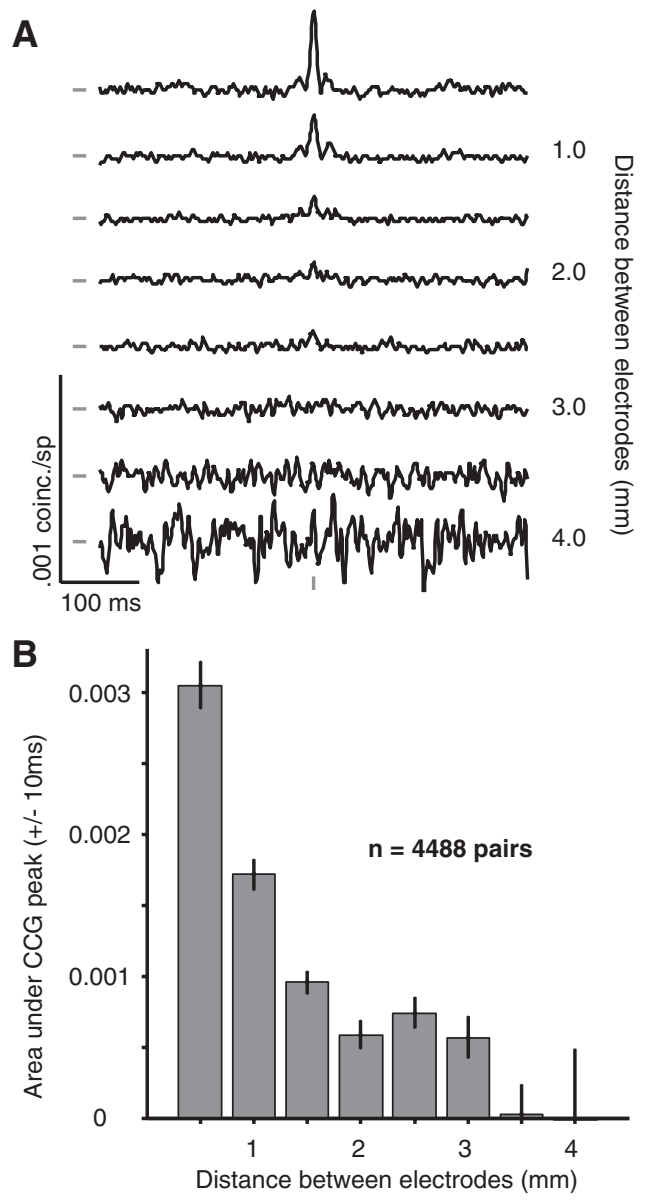

Figure 5. CCGs after removing long time scale correlation. $A$, Average CCGs, after jitter correction, for pairs of neurons grouped by distance. A large peak, centered on zero time lag, is evident at close distances. The increasing noise in the lower plots is due to the smaller number of pairs at those distances. The tick marks to the left of the CCGs indicate a value of 0 coinc./sp. The tick mark at the bottom of the CCGs indicates zero time lag. $\boldsymbol{B}$, The area under the $C(G$ peak, within $\pm 10 \mathrm{~ms}$ of zero, falls off quickly with distance, reaching zero for pairs $>3 \mathrm{~mm}$ apart.

Average jitter-corrected CCGs (jitter window of $50 \mathrm{~ms}$ ), grouped by the distance between electrodes, are shown in Figure $5 \mathrm{~A}$. In these CCGs, a narrow peak centered on zero time lagcapturing the prevalence of synchronous activity — was evident for nearby neurons, $<1.0 \mathrm{~mm}$ distant. At longer distances (1-2 $\mathrm{mm})$ the peak amplitude was substantially smaller. For more distant neurons ( $>3 \mathrm{~mm}$ ), no central peak was detectable. To quantify the strength of synchrony, we integrated the area under the CCG peak from $\pm 10 \mathrm{~ms}$. A histogram of this area is shown in Figure $5 B$ and illustrates the rapid decay of synchrony with distance.

To facilitate a direct comparison between the structure of synchrony and correlated variability, we plot the dependence of synchrony on both distance and tuning similarity in Figure 6 (to be compared with Fig. 3). Figure $6 \mathrm{~A}$ shows the dependence of synchrony on distance for four groups of neurons with different degrees of tuning similarity. In each case, the strength of synchrony declines quickly with distance, reaching values not significantly different from zero within $3.5 \mathrm{~mm}$. To investigate the dependence of synchrony on tuning similarity, we grouped pairs into four distance categories. For the closest pairs $(<1.0$ $\mathrm{mm}$; thick line in Fig. $6 B$ ), synchrony decayed quickly as the tuning of the neurons became increasing different (more negative values of $\left.r_{\text {signal }}\right)$. At intermediate distances $(1.0-2.0 \mathrm{~mm})$,
A
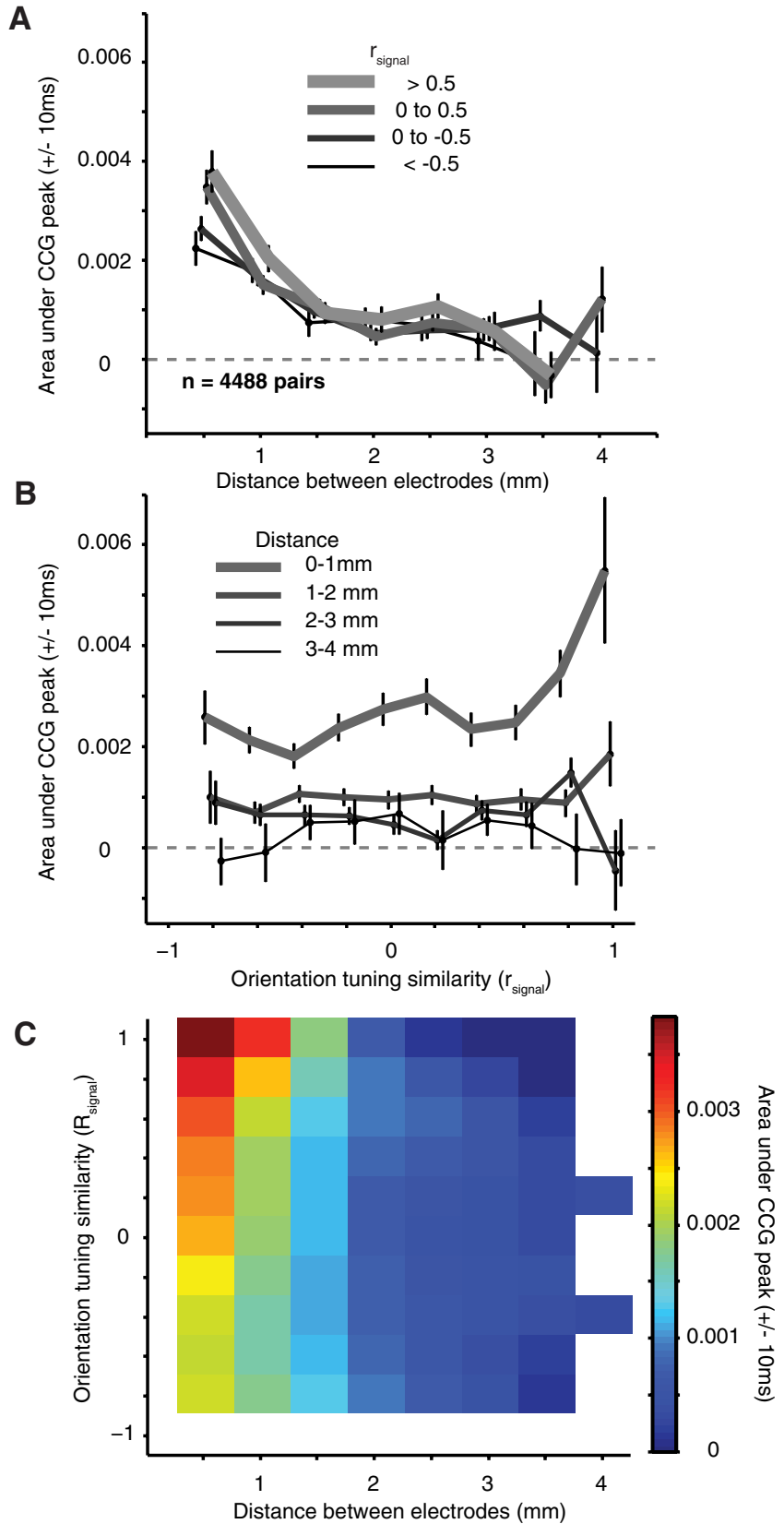

Figure 6. Dependence of synchrony on distance and tuning similarity. $\boldsymbol{A}$, The dependence of synchrony, quantified by the area under the $C(G$ peak, on distance for pairs grouped based on their orientation tuning similarity $\left(r_{\text {signal }}\right.$ thicker lines are for more similar orientation tuning). $\boldsymbol{B}$, The dependence of synchrony on orientation tuning similarity for pairs grouped by distance $\left(r_{\text {signal, }}\right.$ thinner lines are for more distant pairs of neurons). $C$, The relationship of synchrony to both distance and tuning similarity (conventions are as in Fig. 3C).

synchrony was also dependent on orientation tuning similarity but still present in neuronal pairs with widely ranging preferences. For long distances $(2-4 \mathrm{~mm})$ synchrony was weak between all pairs of neurons, regardless of whether their tuning was similar.

Figure $6 C$ shows the full relationship between synchrony, $r_{\text {signal }}$, and distance. Compared with the corresponding figure for $r_{\mathrm{sc}}$ (Fig. 3C), synchrony declined more quickly with distance than $r_{\mathrm{sc}}$; it fell to zero at distances for which $r_{\mathrm{sc}}$ remained significantly above zero. However, synchrony and $r_{\text {sc }}$ showed a similar dependence on $r_{\text {signal. }}$. We fit the synchrony data with the same function used to describe the trends seen in $r_{\text {sc }}$ (Eq. 7) 
and found a much more rapid decay with distance (slope of $1.88 \times 10^{-3}$ coinc $^{*} \mathrm{spk}^{-*} \mathrm{~mm}^{-1} \pm 0.19 \times 10^{-3}$ and a weak dependence on $r_{\text {signal }}$ (decay constant of $2.47 \pm 0.50$ ). With an offset of $0.40 \times 10^{-3} \pm 0.07 \times 10^{-3}$ and an intercept of $4.19 \times 10^{-3} \pm 0.31 \times 10^{-3}$, this function accounted for $95 \%$ of the variance in the data.

The dependence of synchrony on distance and tuning similarity shown in Figure 6 is evident after averaging across a large number of neuronal pairs. It is possible that this obscures the presence of synchrony at long distances in a small number of pairs. We therefore determined for each pair whether the CCG had a significant peak, defined as a bin within $10 \mathrm{~ms}$ of zero lag that exceeded 5 SDs of the noise level (measured from time lags -250 to -200 and $200-250 \mathrm{~ms}$ ). For nearby pairs of neurons $(<0.75 \mathrm{~mm}), \sim 13 \%$ of pairs had significant peaks. The proportion of such pairs fell rapidly: at distances of $>2.0$ $\mathrm{mm}$, it dropped $>15$-fold to $0.75 \%$ (10 of 1333 pairs). The area of the peak for significant CCGs (data not shown) decayed at a similar rate to the value computed across all pairs (Fig. 5B). Thus, we found little evidence for the existence of significant synchrony over long distances $(>2 \mathrm{~mm})$, either among individual pairs or in the population average. It is important to note, however, that these measurements were made with a single type of stimulus (sinusoidal gratings), and synchrony is known to depend on stimulus parameters (Gray and Viana Di Prisco, 1997; Friedman-Hill et al., 2000; Frien et al., 2000; Kohn and Smith, 2005; Zhou et al., 2008). We cannot rule out, therefore, that long-range synchrony may arise for other visual stimuli.

\section{Correlation of spontaneous activity}

A potentially important source of evoked response variability is the influence of ongoing or spontaneous activity, which is highly structured in space and time (Arieli et al., 1996; Tsodyks et al., 1999; Chiu and Weliky, 2001; Kenet et al., 2003; Fiser et al., 2004). Arieli et al. (1996) showed, for instance, that trial-to-trial fluctuations in a voltage sensitive dye signal arise from combining a consistent evoked response with a variable background activity. The degree to which fluctuations in spontaneous activity contribute to shared or independent spiking variability is unclear, however. To address this, we asked whether the spatial scale of correlated spontaneous activity was similar to that of evoked responses, indicating that they are generated by the same circuits.

We recorded spontaneous activity continuously for 15-30 min periods in 6 of the 7 array implants. We divided the data into $1.28 \mathrm{~s}$ "trials," separated by an interval of $1.5 \mathrm{~s}$, to mimic the structure of our evoked data. Because the spontaneous activity was recorded many hours after the evoked activity, we sorted the data independently. Using the same criteria as for the evoked activity, we isolated 166 single neurons from these recordings, providing 2738 pairs. We analyzed the dependence of $r_{\mathrm{sc}}$ on the distance between neurons (Fig. 7A, solid line). The $r_{\mathrm{sc}}$ for spontaneous activity decayed significantly with distance $(r=-0.22$, $p<0.0001)$ falling approximately to half its peak value at a distance of $4 \mathrm{~mm}$ (solid line), a similar decay to that observed for evoked activity. Whereas the spatial structure of correlation was similar between spontaneous and evoked activity, there was a striking difference in its strength: the average $r_{\text {sc }}$ value for spontaneous activity was $0.299 \pm 0.003$, nearly twofold higher than the average correlation of evoked activity $(0.176)$ in our dataset $(p<0.0001)$.

The difference in correlation of spontaneous and evoked ac-
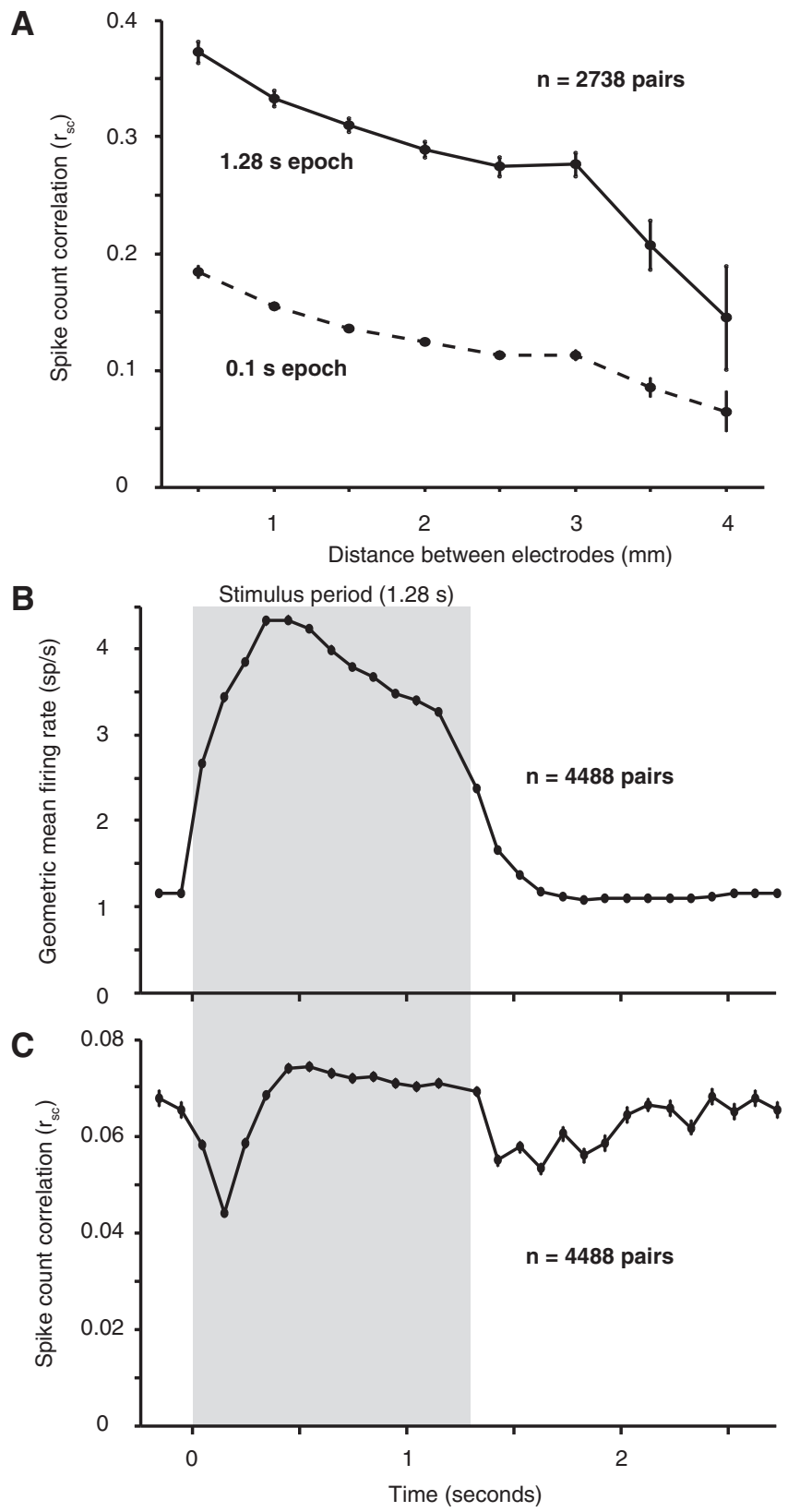

Figure 7. Dynamics of correlation. $A$, In a separate data collection period in 6 of the 7 array implants, we recorded $15-30 \mathrm{~min}$ of spontaneous activity. The value of $r_{\mathrm{sc}}$ for 2738 pairs decreased with distance, but was much higher at all distances than during the evoked response. Using a smaller 0.1 s epoch, the same as in $\boldsymbol{B}$ and $\boldsymbol{C}$, resulted in a lower value for $r_{\text {sc }}$. However, it was still significantly higher than that observed in the ISI after the end of the visual stimulus ( $($ ). $B$, The average geometric mean firing rate across all 12 stimulus directions and all neurons. The stimulus period (1.28 s) is indicated by the gray shaded region, and the nonshaded regions represent the blank interstimulus intervals. Each data point represents the average value for a $100 \mathrm{~ms}$ window centered at the time on the abscissa. $\boldsymbol{C}$, The value of $r_{\mathrm{sc}}$ computed in the same $100 \mathrm{~ms}$ windows as above.

tivity suggests that sensory input can strongly reduce the correlation of ongoing activity. We investigated the dynamics of this process by comparing the correlation in the spontaneous activity immediately preceding and following the presentation of each visual stimulus in our original dataset. Specifically, we divided the stimulus and interstimulus interval (ISI) into $100 \mathrm{~ms}$ response windows, starting $200 \mathrm{~ms}$ before each stimulus and extending through the blank interval until just before the onset of the subsequent stimulus. We combined data from all pairs, regardless of 
their distance, because we observed no dependence of dynamics on spatial separation. We computed the average firing rate in each bin-the population PSTH-which shows the change in firing rate induced by the visual stimulus (Fig. $7 B$, gray box). For each 100 ms epoch, we also computed the value of $r_{\text {sc }}$ (Fig. 7C). At response onset, correlation dropped by $>30 \%$ from $0.065 \pm 0.002$ to $0.044 \pm 0.001$. It slowly rose over the next 400 $\mathrm{ms}$ and then maintained a stable, relatively high level. At stimulus offset, correlation decreased again, followed by a slow rise through the ISI.

The difference between the magnitude of correlation at the end of the ISI and during long periods of spontaneous activity (0.065 vs 0.299$)$ might reflect a slow strengthening during uninterrupted spontaneous activity or simply that correlation measured in brief windows $(100 \mathrm{~ms})$ is weaker than that measured across the duration of the trial (1.28 s). This latter contribution is evident in the weaker $r_{\mathrm{sc}}$ of evoked activity computed in $100 \mathrm{~ms}$ bins (peak of 0.074) compared with that derived from the full stimulus interval (0.176). We therefore calculated the correlation of uninterrupted spontaneous activity in brief epochs $(0.1 \mathrm{~s})$ : the mean $r_{\mathrm{sc}}$ across all distances was $0.138 \pm 0.002$, significantly larger than the value of $0.065 \pm 0.002$ we observed at the end of the ISI and approximately triple the value at response onset (Fig. $7 A$, dotted line). The distance dependence of $r_{\mathrm{sc}}$ measured on this time scale was similar to that measured over $1.28 \mathrm{~s}$.

In summary, we find that the spatial structure of correlated activity is similar for spontaneous and evoked activity, indicating that the two may arise from the same mechanisms and circuits. However, during long periods of uninterrupted spontaneous activity, correlation is approximately twice as strong as during evoked activity. The stimulus-driven reduction in correlation is rapid, and particularly evident at stimulus onset and offset. After the stimulus ends, correlation rises slowly over the $1.5 \mathrm{~s}$ ISI. These results indicate that the processes that lead to correlated variability are altered by inputs to the network, whose effect appears to linger for many seconds after stimulus offset.

\section{Discussion}

We have shown that correlation has a different spatial extent depending on the temporal scale on which it is measured. Precise synchrony extends for $<3 \mathrm{~mm}$, whereas trial-to-trial variability is correlated between cells separated by as much as $10 \mathrm{~mm}$. Correlation on both time scales is strongest for pairs of neurons with similar tuning. The spatial structure of correlated variability is similar during prolonged periods of spontaneous activity, but is nearly twofold larger in magnitude.

\section{Synchrony}

Consistent with our findings, previous studies have also reported that V1 synchrony depends on neuronal separation (Toyama et al., 1981; Michalski et al., 1983; Ts'o et al., 1986; Gray et al., 1989; Schwarz and Bolz, 1991; Hata et al., 1993; Samonds et al., 2006) or similarity of orientation preference (Ts'o et al., 1986; Ts'o and Gilbert, 1988; Engel et al., 1990; Schwarz and Bolz, 1991; Kohn and Smith, 2005). An important distinction between these studies and ours, however, is that previous studies relied on measurements of CCG peak height or area, which can overestimate sharp synchrony by including the influence of a broad CCG peak (as in Fig. $4 F$ ). Our method of calculating the CCG removed correlation on a time scale $>50 \mathrm{~ms}$, which revealed a limited spatial extent of synchrony. In addition, most previous studies were based on recording sequentially from individual pairs and thus contained relatively small datasets. By measuring synchrony between all possible pairings of neurons recorded by the array, we were able to analyze thousands of pairs, providing more accurate and detailed measurements.

In V1, the shared input that generates synchrony could be provided by feedforward input from the LGN (extending $<1$ $\mathrm{mm}$ ), horizontal connections within V1 (extending up to 6 $\mathrm{mm}$ ), and feedback from extrastriate visual cortex (extending $>10 \mathrm{~mm}$ ). The spatial extent of synchrony, and its dependence on tuning similarity, suggests that horizontal connections contribute strongly. These connections target distant ( $>500 \mu \mathrm{m})$ neurons with similar preferences, but appear nonspecific on a more local scale (Gilbert and Wiesel, 1983; Malach et al., 1993; Bosking et al., 1997). Synchrony between nearby cells might thus be expected to be independent of tuning (see also Das and Gilbert, 1999; Maldonado et al., 2000), whereas we found that synchrony is stronger between neurons with similar tuning, even for the most closely spaced neurons recorded $(400 \mu \mathrm{m})$ (see also Kohn and Smith, 2005). This could be because the input provided by distant neurons will be strongest to nearby neurons that have the same preference as the distant source (and therefore each other). Thus, even on a local scale, some common input will depend on the cells' relative preferences. In addition, although it shows little anatomical specificity, local input may have substantial functional specificity, because nearby neurons can have strikingly distinct orientation tuning (Ohki et al., 2006). Finally, the local specificity of synchrony may be generated or modulated by other mechanisms, such as gamma oscillations produced by recurrent excitation and inhibition (Lytton and Sejnowski, 1991; Wang and Buzsáki, 1996; Traub et al., 2001; Hasenstaub et al., 2005; Morita et al., 2008) or gap-junction-coupled networks (Connors and Long, 2004).

Our measurements constrain the functional role of synchrony in visual processing. Specifically, the limited spatial extent we observe suggests synchrony is ill suited for binding the distributed representation of visual objects. Even small visual stimuli activate a spatially extended region of visual cortex: the cortical magnification factor at the eccentricity of our recordings is $\sim 3 \mathrm{~mm} / \mathrm{deg}$ (Van Essen et al., 1984; Tootell et al., 1988). Most visual objects would thus activate neurons separated by distances much greater than the reach of the mechanisms generating synchrony. Of course, other visual stimuli may generate more spatially extensive synchrony. An important test for the binding-by-synchrony hypothesis will thus be to see whether neurons separated by large distances can be precisely synchronized.

\section{Correlated trial-to-trial variability}

Despite the substantial impact of correlated variability on population coding (see below), its properties and the underlying mechanisms have gone largely unexplored. Several studies have shown that $r_{\text {sc }}$ for nearby neurons depends on the similarity of tuning preference (Zohary et al., 1994; Bair et al., 2001; Kohn and Smith, 2005); others have shown that it decays with distance (in motor and parietal cortex; Lee et al., 1998). Our data show that $r_{\text {sc }}$ decays slowly with distance and remains significantly above zero for neurons up to $10 \mathrm{~mm}$ apart; at all distances, $r_{\mathrm{sc}}$ is largest for pairs of neurons with similar orientation preferences. Such spatially extended correlation is consistent with recent electrophysiological (Nir et al., 2008) and 


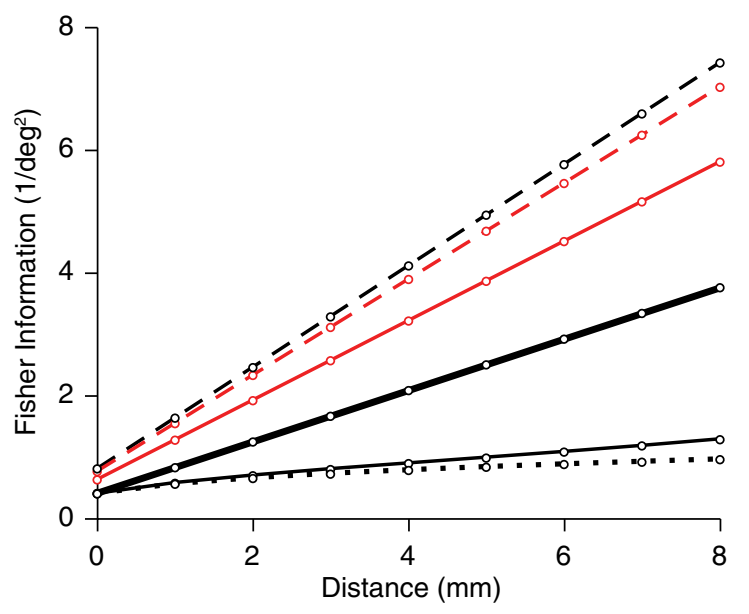

Figure 8. Impact of correlation structure on the Fisher information. We calculated the Fisher information represented by a population of neurons with various correlation structures: no correlation (red line), fixed correlation (red dashed line), correlation that decays with distance but not $r_{\text {signal }}$ (black dashed line), correlation that decays with $r_{\text {signal }}$ but not distance (black dotted line), and the full correlation structure measured in our experimental data, including both the dependence on distance and tuning similarity (thin black line). If $r_{\text {sc }}$ decreased more quickly with distance (at the rate we observed for synchrony), the decrease in the Fisher information would be mitigated (thick black line).

imaging (Fox and Raichle, 2007) data suggesting widespread (e.g., interhemispheric) correlated spontaneous activity.

Horizontal connections may contribute to generating the correlated variability we observe, particularly for distances less than $\sim 6 \mathrm{~mm}$ (Angelucci et al., 2002). Although these connections may also contribute over larger distances-for instance, if slowly fluctuating input is relayed through multiple synapses-a more straightforward interpretation is that feedback connections from extrastriate cortex are involved. Feedback connections extend over $10 \mathrm{~mm}$ (Rockland and Knutson, 2000; Angelucci et al., 2002) and form a major source of input to V1 (Felleman and Van Essen, 1991; Salin and Bullier, 1995). The dependence of $r_{\mathrm{sc}}$ on similarity in orientation preference would require feedback to target neurons with similar preferences, for which there is some anatomical evidence (Angelucci et al., 2002; Shmuel et al., 2005). That correlation may arise from input conveyed via feedback connections is rarely considered in theoretical studies, which typically focus on how correlation affects decoding by downstream neurons. Our results suggest that downstream neurons may in fact be an important contributor to correlated variability.

Correlated variability can be either helpful or harmful to the accuracy of a population code, depending on its properties. How does the correlation structure we observe affect population coding? To address this issue we computed the Fisher information of a population of model neurons, using a number of different correlation structures (see Materials and Methods) (Fig. 8). The Fisher information provides a lower bound on the error of an optimal unbiased decoder and is proportional to the common metric $d^{\prime}$. It thus represents a useful benchmark for comparing the impact of different correlation structures on the accuracy with which a population encodes a variable.

We considered multiple pools of 100 neurons with evenly distributed orientation preferences, separated from each other by $0.5 \mathrm{~mm}$ along one spatial dimension. The red line shows the performance of a population without correlation (i.e., with neurons whose variability is independent). As expected, performance increases linearly with the size of the population or, in our calculations, the extent of cortex activated. Consistent with previous findings (Abbott and Dayan, 1999; Averbeck et al., 2006), fixed correlation (red dashed line) improves the accuracy of the population code, relative to independent neurons, whereas $r_{\text {signal- }}$-dependent correlation (of the form observed in our data) strongly reduces the Fisher information (black dotted line). Including the distance but not $r_{\text {signal }}$ dependence, we observe results in similar performance to fixed correlation strength (black dashed line). This is because the decay with distance simply alters the mean correlation as the population increases but, as it is unrelated to cells' tuning, does not impair the encoding of orientation. Finally, the full correlation structure we measured, including both the dependence on distance and $r_{\text {signal }}$, results in only a slight improvement compared with the $r_{\text {signal }}$ dependence alone (thin black line). Had $r_{\text {sc }}$ decreased more quickly with distance the detrimental effect of correlation would have been mitigated (thick black line, using the more rapid distance dependence observed for synchrony). Our data thus show that the correlation structure in V1 reduces the Fisher information (relative to that provided by independent neurons), even when a spatially extended population is taken into account. It is important to note, however, that the ultimate impact of correlation will depend on the manner in which responses are decoded and that the stimulus dependence of correlation observed on brief time scales may provide an additional source of information (Montani et al., 2007).

\section{Dynamics of correlated variability}

The temporal evolution of synchrony is related to an animal's behavior and to features of the visual stimulus (Vaadia et al., 1995; de Oliveira et al., 1997; Hirabayashi and Miyashita, 2005; Samonds and Bonds, 2005; van der Togt et al., 2006). Our measurements of the dynamics of correlated variability represent, to our knowledge, the first such study. We find that correlated variability drops sharply at both response onset and offset. Response onset has previously been shown to be particularly reliable (Müller et al., 2001; Uka and DeAngelis, 2003). Our data indicate that in addition to being more reliable, neuronal variability is also relatively independent at response onset.

After stimulus offset, correlated variability grows in magnitude. During long periods of spontaneous activity, correlation is approximately twice as strong as during evoked responses and nearly triple that observed at response onset. Previous studies have proposed that sensory input simply sums with ongoing activity (Arieli et al., 1996) or that the dynamics of ongoing activity are hardly affected by sensory input (Fiser et al., 2004). Our results, in contrast, suggest that sensory drive strongly reduces the correlation of spontaneous activity (see also Kohn and Smith, 2005; and Gutnisky and Dragoi, 2008). The elevated correlation in the absence of a stimulus may reflect the default or idle network state, which is disrupted when a subset of neurons in the network is driven by sensory input. Consistent with this idea, nearby neurons in mouse barrel cortex have highly correlated intracellular membrane potentials during quiet wakefulness, which is disrupted during active whisking (Poulet and Petersen, 2008).

\section{Other factors affecting correlation}

In a pair of neurons with fixed common input, correlation increases with firing rate (de la Rocha et al., 2007). In principle, the trends in correlation we observe could thus reflect a change in the common input provided to the pair or simply 
follow from changes in rate. Although we found trends for firing rate to vary with distance and $r_{\text {signal }}$, they were small and not sufficient to explain the change in $r_{\mathrm{sc}}$ (see supplemental Fig. 1, available at www.jneurosci.org as supplemental material). The structure of $r_{\mathrm{sc}}$ that we observe thus arise from changes in the strength of shared input, presumably relayed by the circuits described above.

A second concern is whether the anesthesia used in our recordings affected the correlation we observed. Several lines of evidence indicate that this was not the case. First, we used sufentanil anesthesia, which is widely used in the study of early cortex and for which there is not a single documented discrepancy with data obtained in awake animals (Movshon et al., 2003). This is distinct from the well known ability of other anesthetics, such as ketamine, to alter strongly cortical responsivity and correlation (Greenberg et al., 2008). Second, the value of $r_{\mathrm{sc}}$ for our data matches those based on recordings from awake macaques. For instance, for nearby neurons with similar orientation tuning $\left(r_{\text {signal }}>0\right)$, we found an $r_{\mathrm{sc}}$ of $0.22 \pm 0.03$, similar to that of similarly tuned neurons in area MT/V5 of awake primates (0.19-0.20) (Zohary et al., 1994; Bair et al., 2001). Finally, recent data obtained in awake macaque V1 show a similar distance dependence of correlation to that reported here (Jason Samonds, personal communication). While we cannot rule out some influence of anesthesia, these arguments strongly suggest that the correlation we report is similar to that observed in awake animals.

Finally, in a previous study (Kohn and Smith, 2005), we found that correlation was stimulus dependent: synchrony between nearby cells is significantly stronger for stimuli that drive a pair of neurons well than for those that do not (see also Samonds and Bonds, 2005). In the present study, we used standardized stimuli (i.e., not optimized for each pair) and combined data across conditions. The magnitude of synchrony we observed for nearby cells with this approach is similar to that generated by the least effective stimuli in Kohn and Smith (2005). With standardized stimuli, synchrony is also dependent on stimulus orientation: at all distances at which it was significant, the most effective stimuli evoked the strongest synchrony (supplemental Fig. 2, available at www. jneurosci.org as supplemental material). Similarly, synchrony had a limited spatial extent for activity evoked by either effective or ineffective stimuli. The dependence of correlated variability on distance and $r_{\text {signal }}$ was also similar for effective and ineffective orientations (supplemental Fig. 3, available at www. jneurosci.org as supplemental material).

In Kohn and Smith (2005), we reported that the stimulus dependence of synchrony and correlated variability (measured on extended time scales) was different and suggested the existence of two distinct mechanisms contributing to correlation. The current results show that these two mechanisms are both sensitive to the neurons' relative orientation preferences, but that each has a distinct spatial extent: one generates synchrony over distances of several millimeters; a second generates correlated variability on a slow time scale and extends for at least $10 \mathrm{~mm}$.

\section{References}

Abbott LF, Dayan P (1999) The effect of correlated variability on the accuracy of a population code. Neural Comput 11:91-101.

Angelucci A, Levitt JB, Walton EJ, Hupé JM, Bullier J, Lund JS (2002) Circuits for local and global signal integration in primary visual cortex. J Neurosci 22:8633-8646.

Arieli A, Sterkin A, Grinvald A, Aertsen A (1996) Dynamics of ongoing activity: explanation of the large variability in evoked cortical responses. Science 273:1868-1871.

Averbeck BB, Latham PE, Pouget AP (2006) Neural correlations, population coding and computation. Nat Rev Neurosci 7:358-366.

Bair W, Zohary E, Newsome WT (2001) Correlated firing in macaque visual area MT: time scales and relationship to behavior. J Neurosci 21:1676-1697.

Blasdel GG, Lund JS (1983) Termination of afferent axons in macaque striate cortex. J Neurosci 3:1389-1413.

Bosking WH, Zhang Y, Schofield B, Fitzpatrick D (1997) Orientation selectivity and the arrangement of horizontal connections in tree shrew striate cortex. J Neurosci 17:2112-2127.

Cavanaugh JR, Bair W, Movshon JA (2002) Nature and interaction of signals from the receptive field center and surround in macaque V1 neurons. J Neurophysiol 88:2530-2546.

Chiu C, Weliky M (2001) Spontaneous activity in developing ferret visual cortex in vivo. J Neurosci 21:8906-8914.

Connors BW, Long MA (2004) Electrical synapses in the mammalian brain. Annu Rev Neurosci 27:393-418.

Das A, Gilbert CD (1995) Long-range horizontal connections and their role in cortical reorganization revealed by optical recording of cat primary visual cortex. Nature 375:780-784.

Das A, Gilbert CD (1999) Topography of contextual modulations mediated by short-range interactions in primary visual cortex. Nature 399:655-661.

de la Rocha J, Doiron B, Shea-Brown E, Josić K, Reyes A (2007) Correlation between neural spike trains increases with firing rate. Nature 448:802-806.

de Oliveira SC, Thiele A, Hoffmann KP (1997) Synchronization of neuronal activity during stimulus expectation in a direction discrimination task. J Neurosci 17:9248-9260.

De Valois RL, Albrecht DG, Thorell LG (1982) Spatial frequency selectivity of cells in macaque visual cortex. Vision Res 22:545-559.

Engel AK, König P, Gray CM, Singer W (1990) Stimulus-dependent neuronal oscillations in cat visual cortex: inter-columnar interaction as determined by cross-correlation analysis. Eur J Neurosci 2:588-606.

Felleman DJ, Van Essen DC (1991) Distributed hierarchical processing in the primate cerebral cortex. Cereb Cortex 1:1-47.

Fiser J, Chiu C, Weliky M (2004) Small modulation of ongoing cortical dynamics by sensory input during natural vision. Nature 431:573-578.

Foster KH, Gaska JP, Nagler M, Pollen DA (1985) Spatial and temporal frequency selectivity of neurones in visual cortical areas V1 and V2 of the macaque monkey. J Physiol 365:331-363.

Fox MD, Raichle ME (2007) Spontaneous fluctuations in brain activity observed with functional magnetic resonance imaging. Nat Rev Neurosci 8:700-711.

Friedman-Hill S, Maldonado PE, Gray CM (2000) Dynamics of striate cortical activity in the alert macaque: I. Incidence and stimulusdependence of gamma-band neuronal oscillations. Cereb Cortex 10:1105-1116.

Frien A, Eckhorn R, Bauer R, Woelbern T, Gabriel A (2000) Fast oscillations display sharper orientation tuning that slower components of the same recordings in striate cortex of the awake monkey. Eur J Neurosci 12:1453-1465.

Furukawa S, Middlebrooks JC (2002) Cortical representation of auditory space: Information-bearing features of spike patterns. J Neurophysiol $87: 1749-1762$.

Gawne TJ, Richmond BJ (1993) How independent are the messages carried by adjacent inferior temporal cortical neurons? J Neurosci 13:2758-2771.

Gawne TJ, Kjaer TW, Hertz JA, Richmond BJ (1996) Adjacent visual cortical complex cells share about $20 \%$ of their stimulus-related information. Cereb Cortex 6:482-489.

Gilbert CD, Wiesel TN (1983) Clustered intrinsic connections in cat visual cortex. J Neurosci 3:1116-1133.

Gilbert CD, Wiesel TN (1989) Columnar specificity of intrinsic horizontal and corticocortical connections in cat visual cortex. J Neurosci 9:2432-2442.

Gray CM (1999) The temporal correlation hypothesis of visual feature integration: still alive and well. Neuron 24:31-47, 111-125.

Gray CM, Viana Di Prisco G (1997) Stimulus dependent neuronal oscilla- 
tions and local synchronization in striate cortex of the alert cat. J Neurosci 17:3239-3253.

Gray CM, König P, Engel AK, Singer W (1989) Oscillatory responses in cat visual cortex exhibit inter-columnar synchronization which reflects global stimulus properties. Nature 338:334-337.

Greenberg DS, Houweling AR, Kerr JN (2008) Population imaging of ongoing neuronal activity in the visual cortex of awake rats. Nat Neurosci 11:749-751.

Grinvald A, Lieke EE, Frostig RD, Hildesheim R (1994) Cortical pointspread function and long-range lateral interactions revealed by real-time optical imaging of macaque monkey primary visual cortex. J Neurosci 14:2545-2568.

Gutnisky DA, Dragoi V (2008) Adaptive coding of visual information in neural populations. Nature 452:220-224.

Harrison MT, Geman S (2008) A rate and history-preserving resampling algorithm for neural spike trains. Neural Comput, in press.

Harrison M, Amarasingham A, Geman S (2007) Jitter methods for investigating spike train dependencies. Computational and Systems Neuroscience Abstracts III-17.

Hasenstaub A, Shu Y, Haider B, Kraushaar U, Duque A, McCormick DA (2005) Inhibitory post-synaptic potentials carry synchronized frequency information in active cortical networks. Neuron 47:423-435.

Hata Y, Tsumoto T, Sato H, Hagihara K, Tamura H (1993) Development of local horizontal interactions in cat visual cortex studied by crosscorrelation analysis. J Neurophysiol 69:40-56.

Hirabayashi T, Miyashita Y (2005) Dynamically modulated spike correlation in monkey inferior temporal cortex depending on the feature configuration within a whole object. J Neurosci 24:10299-10307.

Kay SM (1993) Fundamentals of statistical processing: estimation theory. Englewood Cliffs, NJ: Prentice-Hall.

Kelly RC, Smith MA, Samonds JM, Kohn A, Bonds AB, Movshon JA, Lee TS (2007) Comparison of recordings from microelectrode arrays and single electrodes in the visual cortex. J Neurosci 27:261-264.

Kenet T, Bibitchkov D, Tsodyks M, Grinvald A, Arieli A (2003) Spontaneously emerging cortical representations of visual attributes. Nature 425:954-956.

Kohn A, Smith MA (2005) Stimulus dependence of neuronal correlation in primary visual cortex of the macaque. J Neurosci 25:3661-3673.

Lee D, Port NL, Kruse W, Georgopoulos AP (1998) Variability and correlated noise in the discharge of neurons in motor and parietal areas of the primate cortex. J Neurosci 18:1161-1170.

Lytton WW, Sejnowski TJ (1991) Simulations of cortical pyramidal neurons synchronized by inhibitory interneurons. J Neurophysiol 66:1059-1079.

Malach R, Amir Y, Harel M, Grinvald A (1993) Relationship between intrinsic connections and functional architecture revealed by optical imaging and in vivo targeted biocytin injections in primate striate cortex. Proc Natl Acad Sci U S A 90:10469-10473.

Maldonado PE, Friedman-Hill S, Gray CM (2000) Dynamics of striate cortical activity in the alert macaque: II. Fast time scale synchronization. Cereb Cortex 10:1117-1131.

Mazurek ME, Shadlen MN (2002) Limits to the temporal fidelity of cortical spike rate signals. Nat Neurosci 5:463-471.

Michalski A, Gerstein GL, Czarkowska J, Tarnecki R (1983) Interactions between cat striate cortex neurons. Exp Brain Res 51:97-107.

Montani F, Kohn A, Smith MA, Schultz SR (2007) The role of correlations in direction and contrast coding in the primary visual cortex. J Neurosci 27:2338-2348.

Moore GP, Segundo JP, Perkel DH, Levitan H (1970) Statistical signs of synaptic interaction in neurons. Biophys J 10:876-900.

Morita K, Kalra R, Aihara K, Robinson HPC (2008) Recurrent synaptic input and the timing of gamma-frequency-modulated firing of pyramidal cells during neocortical "UP" states. J Neurosci 28:1871-1881.

Movshon JA, Albright TD, Stoner GR, Majaj NJ, Smith MA (2003) Cortical responses to visual motion in alert and anesthetized monkeys. Nat Neurosci 6:3; author reply 3-4.

Müller JR, Metha AB, Krauskopf J, Lennie P (2001) Information conveyed by onset transients in responses of striate cortical neurons. J Neurosci 21:6978-6990.

Nir Y, Mukamel R, Dinstein I, Privman E, Harel M, Fisch L, Gelbard-Sagiv H, Kipervasser S, Andelman F, Neufeld MY, Kramer U, Arieli A, Fried I, Malach R (2008) Interhemispheric correlations of slow spontaneous neuronal fluctuations revealed in human sensory cortex. Nat Neurosci. Advance online publication. Retrieved October 31, 2008. doi:10.1038/nn.2177

Nordhausen CT, Maynard EM, Normann RA (1996) Single unit recording capabilities of a 100 microelectrode array. Brain Res 726:129-140.

Ohki K, Chung S, Kara P, Hübener M, Bonhoeffer T, Reid RC (2006) Highly ordered arrangement of single neurons in orientation pinwheels. Nature 442:925-928.

Perkel DH, Gerstein GL, Moore GP (1967) Neuronal spike trains and stochastic point processes II. Simultaneous spike trains. Biophys J 7:419-440.

Poulet JFA, Petersen CCH (2008) Internal brain state regulates membrane potential synchrony in barrel cortex of behaving mice. Nature 454:881-885.

Reich DS, Mechler F, Victor JD (2001) Independent and redundant information in nearby cortical neurons. Science 294:2566-2568.

Rockland KS, Knutson T (2000) Feedback connections from area MT of the squirrel monkey to areas V1 and V2. J Comp Neurol 425:345-368.

Rousche PJ, Normann RA (1992) A method for pneumatically inserting an array of penetrating electrodes into cortical tissue. Ann Biomed Eng 20:413-422.

Salin PA, Bullier J (1995) Corticocortical connections in the visual system: structure and function. Physiol Rev 75:107-154.

Samonds JM, Bonds AB (2005) Gamma oscillation maintains stimulus structure-dependent synchronization in cat visual cortex. J Neurophysiol 93:223-236.

Samonds JM, Zhou Z, Bernard MR, Bonds AB (2006) Synchronous activity in cat visual cortex encodes collinear and cocircular contours. J Neurophysiol 95:2602-2616.

Schwarz C, Bolz J (1991) Functional specificity of a long-range horizontal connection in cat visual cortex: a cross-correlation study. J Neurosci 11:2995-3007.

Seriès P, Latham PE, Pouget A (2004) Tuning curve sharpening for orientation selectivity: coding efficiency and the impact of correlations. Nat Neurosci 7:1129-1135.

Shadlen MN, Movshon JA (1999) Synchrony unbound: a critical evaluation of the temporal binding hypothesis. Neuron 24:67-77, 111-125.

Shadlen MN, Newsome WT (1998) The variable discharge of cortical neurons: implications for connectivity, computation, and information coding. J Neurosci 18:3870-3896.

Shmiel T, Drori R, Shmiel O, Ben-Shaul Y, Nadasdy Z, Shemesh M, Teicher M, Abeles M (2006) Temporally precise cortical firing patterns are associated with distinct action segments. J Neurophysiol 96:2645-2652.

Shmuel A, Korman M, Sterkin A, Harel M, Ullman S, Malach R, Grinvald A (2005) Retinotopic axis specificity and selective clustering of feedback projections from V2 to V1 in the owl monkey. J Neurosci 25:2117-2131.

Shoham S, Fellows MR, Normann RA (2003) Robust, automatic spike sorting using mixtures of multivariate t-distributions. J Neurosci Methods 127:111-122.

Smith MA, Bair W, Movshon JA (2002) Signals in macaque V1 neurons that support the perception of Glass patterns. J Neurosci 22:8334-8345.

Stettler DD, Das A, Bennett J, Gilbert CD (2002) Lateral connectivity and contextual interactions in macaque primary visual cortex. Neuron 36:739-750.

Suner S, Fellows MR, Vargas-Irwin C, Nakata GK, Donoghue JP (2005) Reliability of signals from a chronically implanted, silicon-based electrode array in non-human primate primary motor cortex. IEEE Trans Neural Syst Rehabil Eng 13:524-541.

Tootell RBH, Switkes E, Silverman MS, Hamilton SL (1988) Functional anatomy of macaque striate cortex. II. Retinotopic organization. J Neurosci 8:1531-1568.

Toyama K, Kimura M, Tanaka K (1981) Organization of cat visual cortex as investigated by cross-correlation technique. J Neurophysiol 46:202-214.

Traub RD, Kopell N, Bibbig A, Buhl EH, LeBeau FEN, Whittington MA (2001) Gap junctions between interneuron dendrites can enhance synchrony of gamma oscillations in distributed networks. J Neurosci 21:9478-9486.

Ts'o DY, Gilbert CD (1988) The organization of chromatic and spatial interactions in the primate striate cortex. J Neurosci 8:1712-1727. 
Ts'o DY, Gilbert CD, Wiesel TN (1986) Relationships between horizontal interactions and functional architecture in cat striate cortex as revealed by cross-correlation analysis. J Neurosci 6:1160-1170.

Tsodyks M, Kenet T, Grinvald A, Arieli A (1999) Linking spontaneous activity of single cortical neurons and the underlying functional architecture. Science 286:1943-1946.

Uka T, DeAngelis GC (2003) Contribution of middle temporal area to coarse depth discrimination: comparison of neuronal and psychophysical sensitivity. J Neurosci 23:3515-3530.

Vaadia E, Haalman I, Abeles M, Bergman H, Prut Y, Slovin H, Aertsen A (1995) Dynamics of neuronal interactions in monkey cortex in relation to behavioural events. Nature 373:515-518.

van der Togt C, Kalitzin S, Spekreijse H, Lamme VA, Supèr H (2006) Syn- chrony dynamics in monkey V1 predict success in visual detection. Cereb Cortex 16:136-148.

Van Essen DC, Newsome WT, Maunsell JHR (1984) The visual field representation in striate cortex of the macaque monkey: asymmetries, anisotropies, and individual variability. Vision Res 24:429-448.

Wang XJ, Buzsáki G (1996) Gamma oscillation by synaptic inhibition in a hippocampal interneuronal network model. J Neurosci 16:6402-6413.

Zhou Z, Bernard MR, Bonds AB (2008) Deconstruction of spatial integrity in visual stimulus detected by modulation of synchronized activity in cat visual cortex. J Neurosci 28:3759-3768.

Zohary E, Shadlen MN, Newsome WT (1994) Correlated neuronal discharge rate and its implications for psychophysical performance. Nature 370:140-143. 